University of Nebraska - Lincoln

DigitalCommons@University of Nebraska - Lincoln

$1-2006$

\title{
Can Environmental Variation Generate Positive Indirect Effects in a Model of Shared Predation?
}

Chad Brassil

University of Nebraska - Lincoln, cbrassil@unl.edu

Part of the Biology Commons

Brassil, Chad, "Can Environmental Variation Generate Positive Indirect Effects in a Model of Shared Predation?" (2006). Faculty Publications in the Biological Sciences. 315.

https://digitalcommons.unl.edu/bioscifacpub/315

This Article is brought to you for free and open access by the Papers in the Biological Sciences at DigitalCommons@University of Nebraska - Lincoln. It has been accepted for inclusion in Faculty Publications in the Biological Sciences by an authorized administrator of DigitalCommons@University of Nebraska - Lincoln. 


\title{
Can Environmental Variation Generate Positive Indirect Effects in a Model of Shared Predation?
}

\author{
Chad E. Brassil ${ }^{*}$
}

Department of Zoology, University of Toronto, 25 Harbord Street, Toronto, Ontario M5S 3G5, Canada; and W. K. Kellogg Biological Station, Michigan State University, Hickory Corners, Michigan 49060

Submitted December 23, 2004; Accepted September 7, 2005;

Electronically published November 7, 2005

Online enhancements: appendixes, video.
Classic apparent competition theory (Holt 1977) predicts a negative, reciprocal indirect effect between two prey via a common enemy; however, population sizes can fluctuate because of the influence of environmental variation, a mechanism labeled here as exogenous. Population sizes can also fluctuate or cycle because of the inherent nature of predator-prey interactions, a mechanism labeled here as endogenous. Endogenously generated cycles have been shown to reduce the strength of negative interactions or even lead to positive interactions (Abrams et al. 1998; Brassil and Abrams 2004), but we understand little about the effect of exogenous variation.

Positive indirect effects have not been documented by empirical studies of long-term dynamics (Chaneton and Bonsall 2000) but in theory can occur via several mechanisms and have been observed in short-term studies (Webster and Almany 2002). Positive indirect effects theoretically can occur because of saturating responses and direct density dependence in the predator (Abrams and Matsuda 1996), egg-limited parasitoids and an egg sink host (Heimpel et al. 2003), or prey that behaviorally reduce foraging rates in response to an increase in predators (Abrams 1987). Finally, an increase in the amplitude of cycles following addition of a second prey species can result in positive indirect effects because of the predator's nonlinear functional response (Abrams et al. 1998). Population fluctuations in the prey reduce the temporal average predator population because a decrease in resources reduces predator growth rate more than an equivalent increase in resources benefits growth (Ruel and Ayres 1999). Positive indirect effects between the prey occur when the addition of the second prey increases the amplitude of variation in the prey populations sufficiently to overcome the potential increase in the predator population caused by increased prey availability.

Previous work has studied the effects of endogenously generated population cycles on the indirect interactions via a shared enemy. Endogenous population cycles are generated by feedbacks within the system such as interactions between predator and prey populations or density dependence within a prey population. Note that demo-

\footnotetext{
* Current address: W. K. Kellogg Biological Station, Michigan State University, 3700 East Gull Lake Drive, Hickory Corners, Michigan 49060; e-mail: brassilc@kbs.msu.edu.
}

Am. Nat. 2006. Vol. 167, pp. 43-54. (C) 2006 by The University of Chicago.

0003-0147/2006/16701-40822\$15.00. All rights reserved.

\begin{abstract}
Classic models of apparent competition predict negative
indirect effects between prey with a shared enemy. If predator per capita growth rates are nonlinear, then endogenously generated pe-
\end{abstract} (eywords: noise amplification, extrinsic forcing, nonlinear dynamics, apparent competition, exogenous variation, subharmonic resonance. 
graphic stochasticity is not considered here because it primarily affects populations at small sizes only. Population fluctuations are common in natural populations (Kendall et al. 1998), but the degree to which these are caused by endogenous or exogenous mechanisms remains uncertain (Ellner and Turchin 1995). Nisbet and Gurney's (1982) early description of environmental variation driving population variation was based on first-order linear approximations. As a result, it was concluded that environmental variation could often be ignored when trying to understand the nature of interspecific interactions. More recently there has been an emphasis on simultaneously examining both endogenous and exogenous variation (Abrams 2004; Coulson et al. 2004).

Time averaging (Puccia and Levins 1985) of simple models suggests that the variances and covariances of the population sizes across time can be important in determining the nature of indirect effects. If variance in population size alone determines indirect effects, then the indirect effects in cycling population may not be sensitively dependent on the mechanism generating the cycles (Holt and Lawton 1993). Similar effects should arise from exogenous or endogenous mechanisms, provided they lead to similar variances and covariances of population sizes across time. However, the nature of endogenous cycles and exogenous cycles may differ so that exact equality of these variances and covariances is unlikely. The amplitude, shape, or period of either endogenous or exogenous cycles may preclude generalizations across mechanisms.

I examine the sensitivity of indirect effects between prey to environmental variation in parameters of a one predator-two prey model with the goal of determining the relative effect of variation in each parameter on the magnitude and direction of the indirect interaction. In addition, I show the importance of nonlinearity in the per capita growth rate of the predator in determining sensitivity of indirect effects to environmental variation. While nonlinear per capita growth rates have been shown to be important in many ecological systems, the specific predictions regarding indirect effects under shared predation are unknown. An important question is whether environmental variation affecting demographic traits can generate sufficient variation so that otherwise negative indirect effects between prey become positive indirect effects. I heuristically describe the mechanisms that underlie the changes in indirect effects caused by exogenous variation.

\section{Model}

Deterministic exogenous variation was examined using a standard predator-prey model with two prey populations:

$$
\begin{aligned}
\frac{d N_{1}}{d t} & =r_{1} N_{1}\left(1-\frac{N_{1}}{k_{1}}\right)-f_{1}\left(N_{1}, N_{2}\right) P, \\
\frac{d N_{2}}{d t} & =r_{2} N_{2}\left(1-\frac{N_{2}}{k_{2}}\right)-f_{2}\left(N_{2}, N_{1}\right) P, \\
\frac{d P}{d t} & =g_{1}\left(N_{1}, N_{2}\right) P+g_{2}\left(N_{2}, N_{1}\right) P-\delta\left(P, N_{1}, N_{2}\right) P .
\end{aligned}
$$

The maximum growth rate of prey $i$ is $r_{i}$, the carrying capacity is $k_{i}$, the functional response of the predator to prey $i$ is $f_{i}$, the numerical response of the predator to prey $i$ is $g_{i}$, and the predator per capita death rate is $\delta$. A dimensionless version of the model is not presented here because of the problem with interpreting the results when examining variation in a scaled parameter. Similar models to equation (1) are well understood in other contexts and can produce positive indirect effects when variation is driven by endogenous mechanisms in the presence of a nonlinear functional response (Abrams et al. 1998). In order to simplify the analysis, I first examined cases in which the two prey were characterized by identical demographic parameters $\left(r_{1}=r_{2}, k_{1}=k_{2}\right.$, etc. $)$ and environmental variation in a parameter affected each prey population simultaneously. This simplification can produce the same results as doubling the carrying capacity of the prey, although with separate dynamical equations for each prey population, the population sizes need not be identical.

\section{Linear Functional Response and Density- Independent Death Rate}

For this model, the functional response increases in proportion to available prey, $f_{i}\left(N_{i}, N_{j}\right)=a_{i} N_{i}$. The predator's attack rate on prey $i$ is $a_{i}$. The per capita birth rate is a linear function of the functional response, $g_{i}\left(N_{i}, N_{j}\right)=$ $c_{i} f_{i}\left(N_{i}, N_{j}\right)$, where the conversion efficiency of captured prey of species $i$ to new predators is $c_{i}$. The per capita death rate of the predator is a constant, $\delta\left(P, N_{1}, N_{2}\right)=$ $m$. See an article by Rinaldi et al. (1993) for a complete description of dynamics with seasonal forcing in the one prey-one predator version of this model.

\section{Nonlinear Model}

Relatively linear predator per capita growth functions eliminate the possibility of certain types of indirect interactions between prey, but highly nonlinear functions in the per capita growth rates often coincide with unstable dynamics. Analysis has been restricted to parameters for which dynamics are stable so that changes in indirect effects can be attributed to exogenous variation. In order to 
show the effect of a highly nonlinear model, I consider a model characterized by a very nonlinear predator growth function that is the result of a consumption-dependent death rate.

The nonlinear model considered here includes a nonlinear functional response and a predator death rate that is dependent on consumed prey. The saturating functional response used here is $f_{i}\left(N_{i}, N_{j}\right)=a_{i} N_{i} /\left(1+h a_{1} N_{1}+\right.$ $h a_{2} N_{2}$ ). This simplified model illustrates the importance of prey dependence in the death rate by shifting all prey dependence from the predator's birth rate to the death rate (Abrams 1997). The predator birth rate is a constant, $g_{i}\left(N_{i}, N_{j}\right)=b_{i}$, and prey consumption is instead incorporated into the predator death rate, $\delta\left(P, N_{1}, N_{2}\right)=$ $m /\left[f_{1}\left(N_{1}, N_{2}\right)+f_{2}\left(N_{2}, N_{1}\right)\right]$. Previous work (Abrams 1997) has suggested that similar nonlinear death rates can greatly alter the effect of increased productivity on predator density in a one predator-one prey system, and the addition of a second prey species has many parallels with increased productivity of a single prey species.

Environmental variation was modeled as a deterministic sine function in one of the demographic parameters. The standardized amplitude is a fraction times the mean value. Analysis initially was restricted to parameters that led to a stable equilibrium, and the amplitude of variable parameters was chosen so that if the parameter were fixed at any value within that range, the system would have a stable equilibrium. This restriction ensured that variation in the population was a result of exogenous variation alone and not a result of exogenous variation moving parameters into a range in which endogenous variation was generated.

More realistic environmental variation also was examined by combining sine functions of different frequencies. A specified number of components were randomly drawn from a uniform distribution of frequencies across a set range. The relative amplitude of each component was based on pink noise (Vasseur and Yodzis 2004), 1/f, where $f$ is the frequency of the component.

Indirect effects between prey populations were quantified by comparing the mean prey population size across time in the presence and in the absence of the other prey. The average across time is represented by angle brackets, with the subscript denoting the populations included in the model when calculating the average:

$$
\text { indirect effect }=\frac{\left\langle N_{i}\right\rangle_{P, N_{i}, N_{j}}-\left\langle N_{i}\right\rangle_{P, N_{i}}}{\max \left(\left\langle N_{i}\right\rangle_{P, N_{i}, N_{j}},\left\langle N_{i}\right\rangle_{P, N_{i}}\right)} .
$$

Dividing the difference by the maximum of either value standardizes the indirect effect between -1 and 1 , which is appropriate for comparing effects that may be positive or negative. The standardized indirect effect can be inter- preted as a fractional change in population size produced by introducing a second prey population. The mean population size is calculated by numerically solving the system using Mathematica 5.0, allowing sufficient time for the system to reach an asymptotic attractor, and then averaging densities across a long time span.

\section{Results}

\section{Linear Functional Response and Density- Independent Death Rate}

For this model, the standardized indirect effect for identical prey (eq. [2]) is always -0.5 when populations reach a stable equilibrium in the absence of variation (app. A in the online edition of the American Naturalist). In other words, the addition of a second prey population reduces the density of the original prey population by a factor of 0.5 because of an increase in the predator population.

The indirect effect between the prey populations is not altered by synchronous variation in a number of parameters for the linear model. Temporal variation in parameters that directly and equally affect both prey populations' growth rates $(r)$ or carrying capacities $(k)$ do not change the average indirect interaction between the prey (app. A, case 1). Predator and prey populations fluctuate with time, and the mean predator population can be altered, but the mean prey population sizes remain the same as the equilibrium population sizes. The predator population responds to changes in prey population sizes in such a way as to buffer the average prey size against environmental variation. Moreover, the indirect effect is unchanged when environmental variation affects the density-independent death rate of the predator, $m$. Variation in $r, k$, or $m$ does not alter the indirect effect because none of these parameters has a nonlinear relationship in the per capita growth rate of the predator.

In contrast, variation in the predator conversion efficiency $c$ or the attack rate $a$ can alter the average indirect effect between prey (fig. 1). The magnitude and direction of the change in the indirect effect depends on the period of the forcing function (the period of environmental variation). The period of the environmental variation determines the covariance that develops between the prey and the variable parameter. For variation in $c$, the indirect effect is a function of that covariance,

$$
\text { indirect effect }=\frac{(-1 / 2)+(a / m)\left(C_{2}-C_{3}\right)}{1-(a / m)\left(C_{2}\right)} \text {, }
$$

where $\left\langle N_{1}\right\rangle>\left\langle N_{2}\right\rangle, C_{2}$ is $\operatorname{Cov}\left(c, N_{1}\right)$ in the absence of prey 2 , and $C_{3}$ is $\operatorname{Cov}\left(c, N_{1}\right)$ with all three species present (app. A, case 3). A similar relationship describes the indirect 
a)

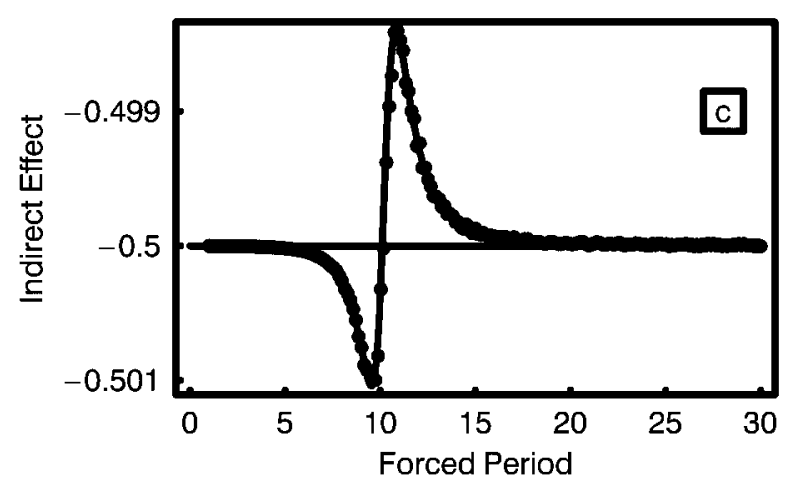

b)

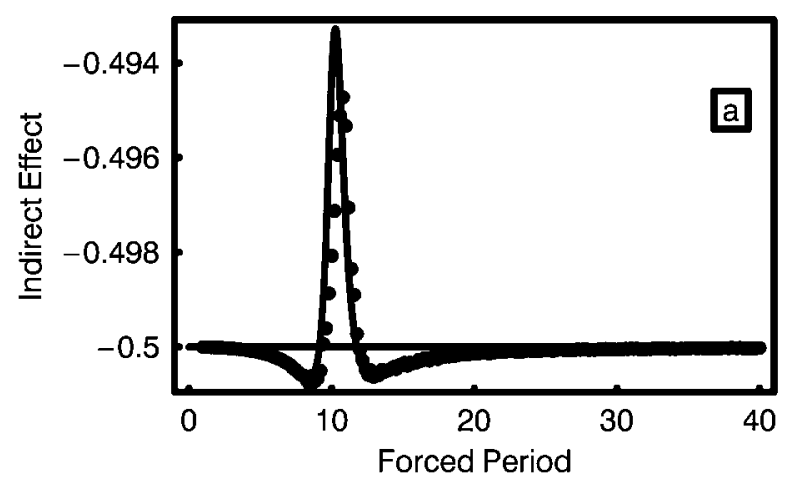

c)

large amplitude

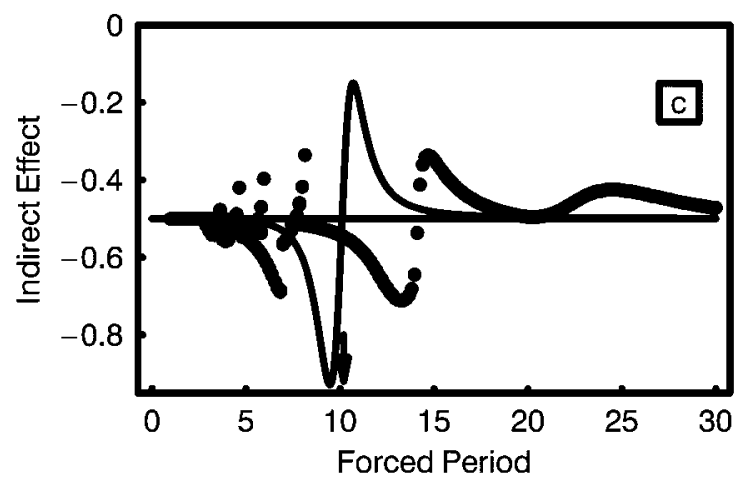

d)

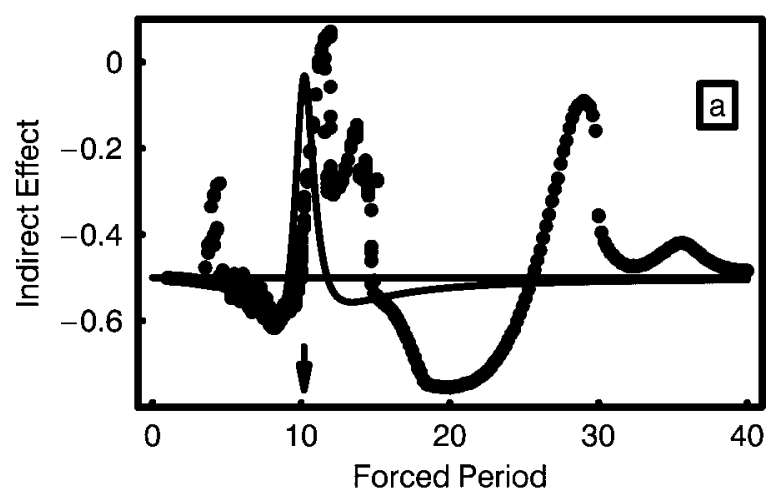

Figure 1: Effect of environmental variation on the indirect effect in conversion efficiency $c$ of consumed prey to predators (panels $a, c$ ) and the attack rate $a$ for a model with a linear functional response and a density-independent death rate for the predator (panels $b$, $d$ ). Results are plotted for small-amplitude environmental variation, $5 \%$ of the mean, and large-amplitude environmental variation, $80 \%$ of the mean. The average indirect effect is plotted versus the period of sinusoidal variation. The solid line is the predicted average indirect effect based on the simplifying assumptions of the transfer function (app. A in the online edition of the American Naturalist). Points represent numerical integration of the full model, repeated 25 times at each period with random starting conditions. Multiple points at the same period illustrate alternative attractors. In the absence of environmental variation, the standardized indirect effect between the prey is -0.5 , and the natural period is 10.2 , as indicated by the arrow. At periods greater than that displayed, indirect effects remain close to -0.5 . Densities for the indirect effect are averaged from $t=500$ to $t=2,500$. The parameters are $r=2.0, k=1.0, \bar{a}=4.0, \bar{c}=0.5$, and $m=0.2$.

effect for environmental variation in the attack rate (app. A, case 4).

The indirect effect is a function of changes in the covariance between the environmentally influenced parameter and the population size, and an analytical approximation to the covariance can be derived using assumptions of a transfer function (Nisbet and Gurney 1982). When parameters in this model lead to a stable equilibrium in the absence of exogenous fluctuations, then the transient dynamics approaching that equilibrium typically are damped oscillations with a period called the natural period of the system. When the period of the driving environmental variable is near the natural period of the transient dynamics, the environmental variation can be magnified by the biological system in a manner similar to resonance in a physical system (Nisbet and Gurney 1982). Smallamplitude environmental fluctuations can result in largeamplitude fluctuations in the model (e.g., Dushoff et al. 2004). A transfer function describes the expected amplitude of fluctuations in the population sizes that results from environmental variation in a parameter due to simple resonance that would occur in a linear system. In a nonlinear model, this assumption is accurate only for smallamplitude environmental variation; nonlinear phenomena such as subharmonic resonance (described below) become important for large-amplitude variation.

The expected indirect effect based on the transfer function is illustrated in figure 1 (solid line). The transfer func- 
tion is a good match at small-amplitude variation (fig. $1 a$, $1 b)$, so I describe this pattern heuristically. First, the indirect effect is altered only at periods near the natural period because that is where the greatest resonance occurs. For variation in $c$ at periods shorter than the natural period, the covariances between $c$ and $N_{1}$ are positive, and the addition of a second prey reinforces the negative indirect effect. The opposite occurs at periods longer than the natural period. For variation in $a$, the covariances between $a$ and $N_{1}$ remain negative on each side of the natural period, and the addition of a second prey reduces the strength of the negative indirect effect. These general patterns are translated to higher periods as the amplitude of environmental variation is increased (app. B in the online edition of the American Naturalist). Large-amplitude variation in a nonlinear system can result in complex resonance patterns in population maxima that are known as "breaking wave" patterns (see Greenman et al. 2004). Adding a second prey is effectively stretching the resonance patterns (app. B), and because the resonance patterns are complex, the addition of a second prey can lead to a wide range of outcomes.

Differences in subharmonic resonance between the twospecies and three-species system are an important nonlinear phenomenon that helps explain the pattern of indirect effects at low periods of environmental variation for largeamplitude variation. In a nonlinear system, resonance can drive the population of any of the species to lower sizes and lead to subharmonic resonance in which the population has maxima at an integer multiple of the environmental period (Nisbet and Gurney 1982). Subharmonic resonance differences occur in at least one of the attractors for much of the left-hand side of figure $1 c, 1 d$. For example, the indirect effect actually becomes positive at periods near 11.5 in figure $1 d$ because the addition of the second prey increases the period of cycles in the predator by subharmonic resonance (fig. 2). The predator population in a system with a single prey cycles at a period equal to the environmental variation, but the predator population in a two-prey system cycles at a period two times that of the environmental variation. Subharmonic resonance causes attack rate minima to occur at one-third and two-thirds of the predator's period instead of a single minimum at halfway through the predator cycle. This shift alters the covariance between $a$ and $N_{1}$, resulting in decreased predation pressure and an increase in prey densities.

It should be noted that for some parameter values, exogenous variation induced chaotic dynamics in population densities, for example, the broad smear of points around periods 4-9 in figure $1 b$. While the interpretation of the effect of variation may be similar to nonchaotic cases, it can be more difficult to ascribe the results to intuitive interactions between the environmental variation and the biological system. For example, chaotic cases can qualitatively exhibit the same subharmonic features of figure 2 , but the height of the peak and time period between peaks will vary such that predator peaks may not always align with peaks in the attack rate $a$. However, the analytical expressions that describe the indirect effect as a function of the covariance in the presence and absence of the second prey are still true.

\section{Multiple Affected Parameters and Nonidentical Prey}

In a natural environment, multiple parameters would probably be influenced by changes in the environment. Here, I describe one such scenario for which the combined variation in two parameters leads to nonadditive results that can be interpreted biologically. Simultaneous variation in the prey growth rate $r$ and in the conversion efficiency $c$ results in a very similar pattern of change in the indirect effect with cycle period as does variation in $a$ alone. Timeaveraging analysis of variation in $r$ and $c$ (app. A, case 5) shows that the determining factor of the indirect effect is still $\operatorname{Cov}\left(c, N_{1}\right)$, the same as for variation in $c$ alone (app. A, case 3 ). However, variation in $r$ can alter the covariance between $c$ and $N_{1}$. This is most apparent when the phase of variation in $r$ and $c$ is offset by one-half of a cycle. In a manner similar to systems with variation in $a$, a difference in subharmonic resonance leads to prey population peaks that coincide with maximum values in $r$ only in the presence of the second prey population. The above results seem to suggest that indirect effects can be altered to a greater extent by environmental variation that alters the per capita growth rates of both prey and predator populations than by environmental variation that affects only the per capita growth rate of one of the two trophic levels.

If the periodic variation examined here represents seasonal variation, it is probable that prey will experience beneficial environments at the same time. However, it is conceivable that each prey experiences optimal growth rates at different times in the season, for example, a prey adapted to the wet season versus a prey adapted to the dry season. This is modeled by shifting the sine functions so that the growth rate of one prey is at a maximum when the growth rate of the other prey is at a minimum. When the period of environmental variation is greater than two times the natural period of the system, the predator peak associated with one peak in prey density begins to strongly affect the next predator peak. The predator population builds up to very high levels after the second of two successive maxima in the two prey populations followed by a very deep crash in the predator population. As a result, the indirect effect can become asymmetric, more negative for one prey population and less negative for the other population. An alternative attractor characterized by sym- 


\section{1-predator-2-prey}

a)

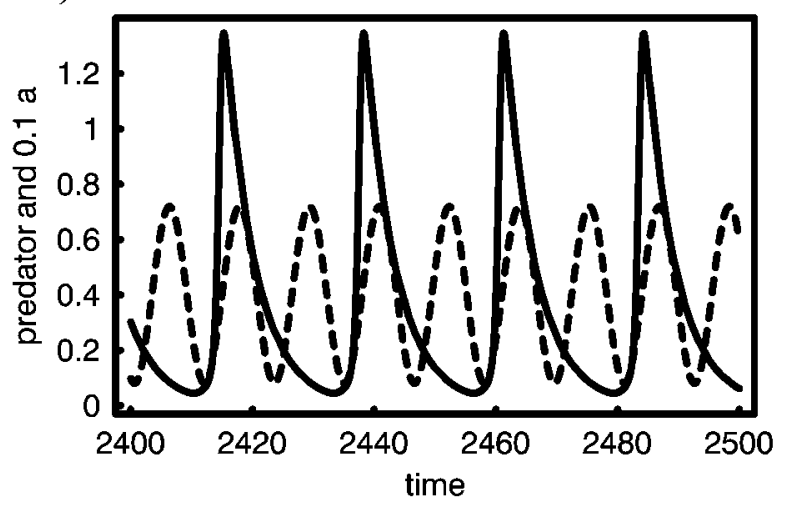

b)

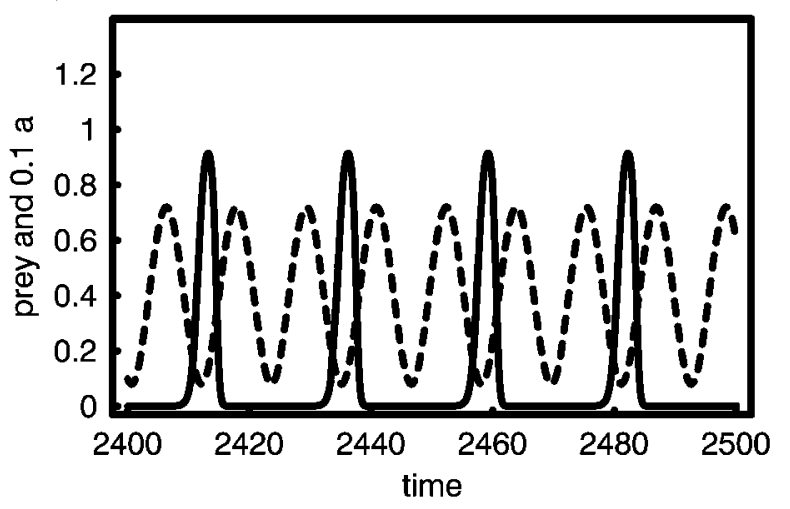

1-predator-1-prey

c)

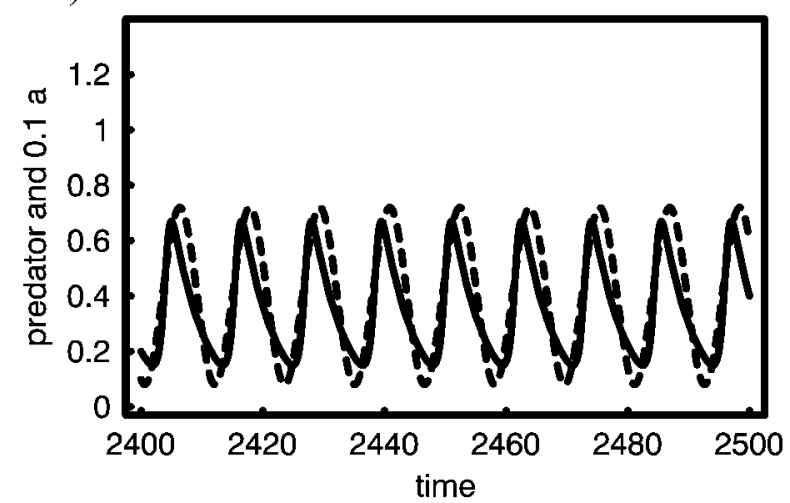

d)

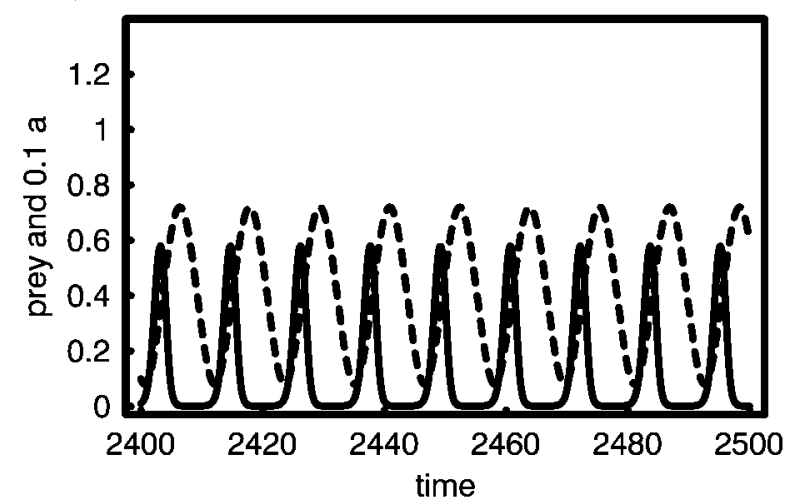

Figure 2: Population dynamics of a system with linear predator responses and periodic variation in the predator's attack rate on both prey. The population size and scaled attack rate are plotted versus time. A positive indirect effect (with a standardized magnitude $=0.043$ ) between prey populations occurs in this case. The parameter $a$, driven by exogenous variation with a period of 11.5 and standardized amplitude of 0.8 , is shown by the dashed line plotted at a scale of $1 / 10$. Only a single prey population is plotted for the one predator-two prey system in $b$. No alternative attractors were observed for this parameter set across 100 random chosen initial starting densities. The parameters are $r=2.0, k=1.0, \bar{a}=4.0$, $c=0.5$, and $m=0.2$.

metric interactions also exists, but a limited set of initial densities lead to the alternative attractor.

In the previous section, each prey species had identical mean demographic values in order to simplify analysis. Environmental fluctuations still modify indirect effects via similar mechanisms when the two prey populations differ in basic demographic traits. In a stable environment, nonidentical prey parameters lead to a reduction in the magnitude of one indirect effect and an increase in the other (app. A, eq. [A18]). Environmental fluctuations modify this indirect effect in a similar manner to the case of identical prey. For example, in the case of different attack rates, figure $1 b$ is translated up for the species with the smaller attack rate just as the indirect effect experienced by that species decreases in the absence of variation. The fundamental pattern of resonance remains, although the degree to which the indirect effect increases or decreases is modified. One surprising result is that environmental variation tends to reduce differences in indirect effects due to nonidentical parameters. For example, when prey differ in their growth rates, environmental cycles in the growth rate of the prey populations often result in an indirect effect closer to -0.5 than in the absence of environmental fluctuations. Environmental fluctuations tend to reduce the asymmetrical indirect effects that result from nonidentical prey.

\section{Nonlinear Model}

In previous work (e.g., Abrams et al. 1998), endogenous cycles have been shown to change indirect effects via a predator because of nonlinearity in the predator's per cap- 
ita growth rate as a function of prey densities. This nonlinearity was absent in the above model. In the linear models, variation in the predator's mean population size does not directly alter the indirect effect between prey. Instead, indirect effects are altered by covariances between environmental parameters and population sizes. In this section, I examine a model with a nonlinear per capita growth rate of the predator (eq. [1], nonlinear version). Analytical expressions of time averages are long and cumbersome for models with nonlinear per capita growth rates (not shown), but they do reveal that variation in population sizes directly alters indirect effects. Consequently, the strength of the indirect effect is now sensitive to variation in all parameters, at least to a small degree.

The indirect effect in the nonlinear model is very sensitive to environmental variation in all parameters except handling time $h$ (fig. 3). For example, in-phase variation in prey growth rates $r$ can lead to large increases or decrease in the indirect effect between prey dependent on the amplitude and period of environmental variation (fig. 4). Some of the largest positive effects occur at periods very similar to those of the largest negative effects because of the peculiarities of resonance. Not all parameter sets exhibit such extreme modifications of indirect effects as illustrated in figure 4, but environmental variation does alter indirect effects in many combinations that retain highly nonlinear per capita growth rates. A model in which predator birth rates depend on consumed prey and death rates are density independent also is sensitive to variation in all the parameters, although to a lesser degree than the model described here (C. E. Brassil, unpublished data).

The indirect effects illustrated above are the result of a single sinusoidal frequency; more realistic environmental variation also can result in large modifications of the indirect effect. The results of sinusoidal environmental variation in $r$ (fig. 3, panel $r$ ) was reexamined by adding a background of pink noise to the dominant forcing period (fig. $5 a$ ). The pattern of the relationship between the dominant period of environmental variation and the indirect effect was very different from the case of pure sinusoidal variation; however, large modifications of the indirect effect still occur.

Multicomponent environmental variation without a dominant period can still modify indirect interactions. In figure $5 b$, I examined pink noise with increasing numbers of components. Environmental variation that consisted of multiple components had a similar average indirect effect as pure sinusoidal variation (one component). As well, increasing the number of components reduced the variation around the mean. On average, multicomponent environmental variation often has effects that are similar to the mean effect of individual components, but that is not true for all situations. For example, a haphazard collection of 10 periods across figure 4 at amplitude 0.8 has an average indirect effect of 0.07 when examined in isolation. However, the net indirect effect is 0.24 when they are equally weighted by amplitude in a combined function of environmental variation. Eliminating the frequency that resulted in the largest positive indirect effect in isolation increased the combined indirect effect from the remaining nine frequencies to 0.34 . Environmental variation composed of multiple frequencies is not necessarily additive. Large nonintuitive modifications of indirect effects still occur when more complex, realistic environmental variation is examined.

\section{Discussion}

The results presented here show that, like endogenous variation, exogenous variation can be very important in determining the direction and the strength of indirect interactions in simple food webs. However, exogenous variation can increase or decrease indirect interactions in both linear and nonlinear models. The response depends on the nature of the variation, resonance with the natural period of any transient dynamics in the underlying system (with no variation), and details of the nonlinearities of the biological system. The sign and magnitude of indirect effects can be greatly altered by environmental variation in all parameters when the predator's numerical response is nonlinear. Complex environmental variation can also generate altered indirect interactions. The results presented here emphasize the importance of accounting for environmental variation when examining empirical systems of apparent competition. If one is studying a system in which environmental fluctuations result in fluctuations in population sizes, then it is probable that the indirect effects will be different than those predicted from equilibriumbased theory.

The first model analyzed here had per capita growth rates that were linear functions of densities, and environmental variation could still alter the indirect effect when variation affected certain parameters. The indirect effect was insensitive to three types of environmental variation in parameters: in-phase variation that affected both prey growth rates, both prey carrying capacities, or the predator death rate. Only variation in parameters directly related to the predator-prey interaction alters the indirect effect. Effectively, the predator per capita growth rate becomes nonlinear with respect to time when the conversion efficiency or the attack rate, as well as prey density, vary with time. At periods near the natural period of the predatorprey interaction, variation in the conversion efficiency and the attack rate led to large decreases or large increases in the indirect effect, depending on the precise period of the forcing. The attack rate and the conversion efficiency are 

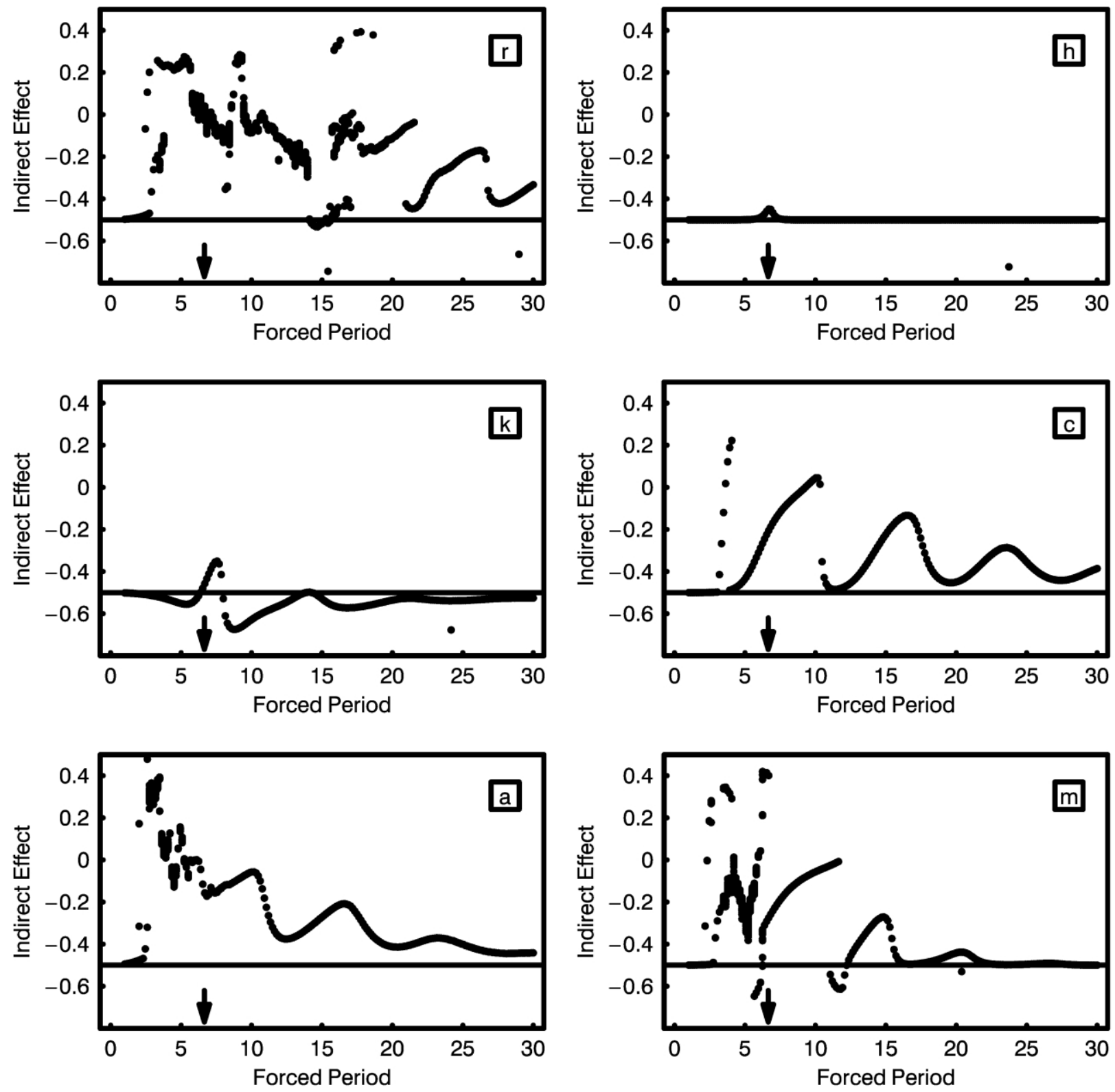

Figure 3: Effect of environmental variation in each parameter on the indirect interaction for the nonlinear model. The average indirect effect is plotted versus the period of sinusoidal variation of a specified period for variation in each parameter $r, h, k, c, a$, and $m$. Points represent numerical integration, repeated 25 times at each period with random starting conditions. In the absence of environmental variation, the standardized indirect effect between the prey is -0.5 and the natural period is 6.65 , as indicated by the arrow. Environmental variation for each plot has a standardized amplitude of 0.8 . The parameter affected by environmental variation is labeled in each plot. For $r$ and $c$, environmental variation at periods greater than 30 results in the same recurring pattern as for periods approaching 30 but with reduced magnitude so that eventually all indirect effects are close to -0.5 . For all other parameters, environmental variation at periods greater than 30 results in indirect effects close to -0.5 . Densities for calculating the indirect effect are averaged from $t=500$ to $t=2,500$. The parameters are $r=2.0, k=1.0, a=2.0, h=0.1, c=0.5$, and $m=0.15$.

affected by characteristics of both the predator and the prey. For example, prey may have special predator avoidance tactics such as cryptic coloration that change in effectiveness based on environmental conditions. In the case of plants as prey, conversion efficiency could vary because of changes in nutrient availability that result in changes in defensive compounds.

In the simple predator-prey model with a linear func- 

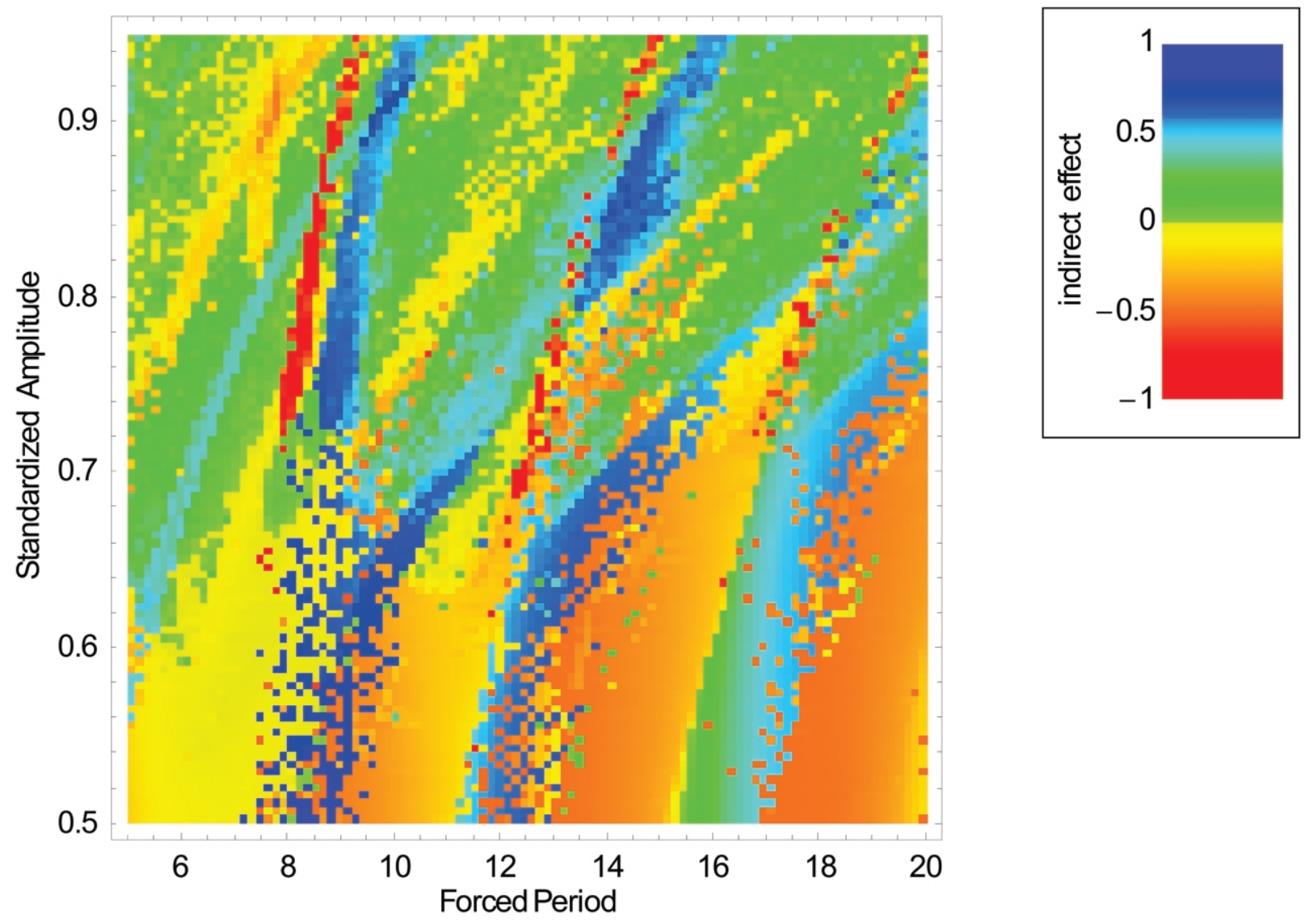

Figure 4: Indirect effect between prey populations for the nonlinear model. The average indirect effect is plotted for a grid of amplitude versus the period of sinusoidal variation in the prey growth rate $r$. The maximum and minimum indirect effects shown in the graph are 0.80 and -0.87 , respectively. In the absence of environmental variation, the indirect effect between the prey is -0.5 , and the natural period measured after 500 time units was 4.6. The numerical integration spanned 2,500 time units with effects calculated by averaging over the last 2,000 time units. The initial densities of predator and prey are random numbers between 0 and 1 for each point on the graph. The parameters are $\bar{r}=2.0, k=1.0, a=5.0$, $h=0.1, c=1.0$, and $m=0.3$.

tional response, the conditions under which changes in the sign of the indirect effects can occur due to environmental variation are somewhat restricted. First, environmental variation has to have a large effect on the magnitude of the demographic parameter. Positive effects were observed only when the standardized amplitude of environmental variation was at least $60 \%$ (between at least 1.6 and 0.4 times the mean demographic parameter). Second, the period of the environmental variation has to be near the natural frequency of the population dynamics or a multiple of that period and the resonant frequency must dominate the environmental variation. Third, transient cycles of the system must be only weakly damped so that they can easily be amplified by environmental variation. In other words, the real part of the dominant eigenvalue of the Jacobian matrix must be near zero, and it must have a nonzero imaginary part. This is more likely to occur near the boundary between stable and unstable dynamics (Greenman and Benton 2003). Positive indirect effects in the linear system require these specific conditions, but modified negative indirect effects occur over a broad range of periods of amplitudes. These restrictions are relaxed in the nonlinear models, for which positive indirect effects occur over a wider range of periods and amplitudes.

Interestingly, there is no general prediction about whether environmental variation greater than or less than the natural period will result in the greatest alteration of the indirect effect. For variation in $a$ of the linear model, positive indirect effects occur when the period of environmental variation is longer than the natural period of the system. When there is variation in $r$ and $c$ of the linear model that is offset in phase by a half-cycle, positive in- 
a)

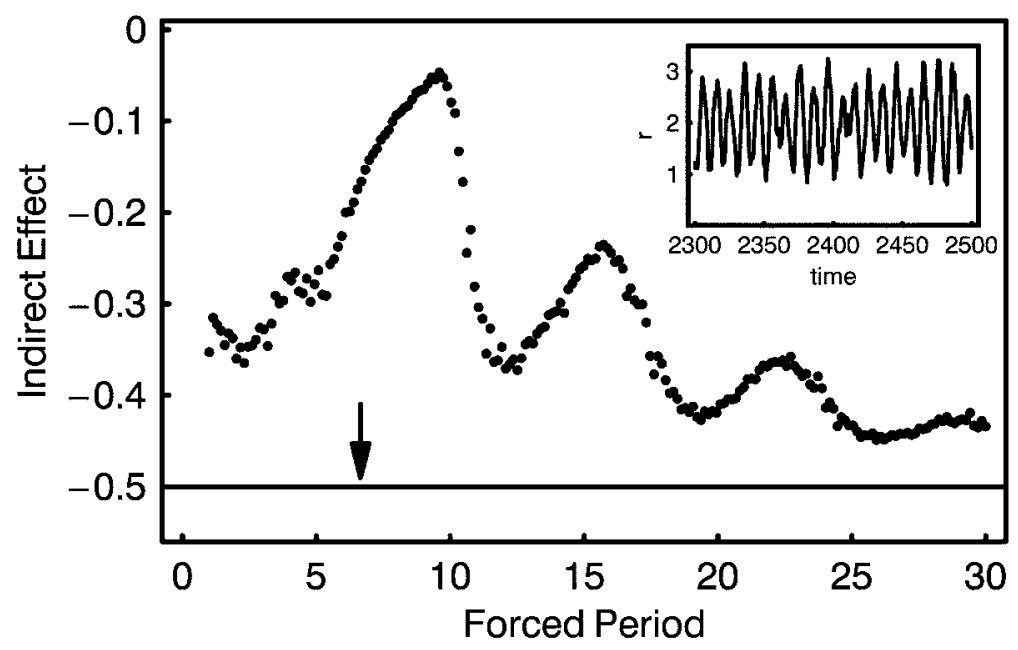

b)

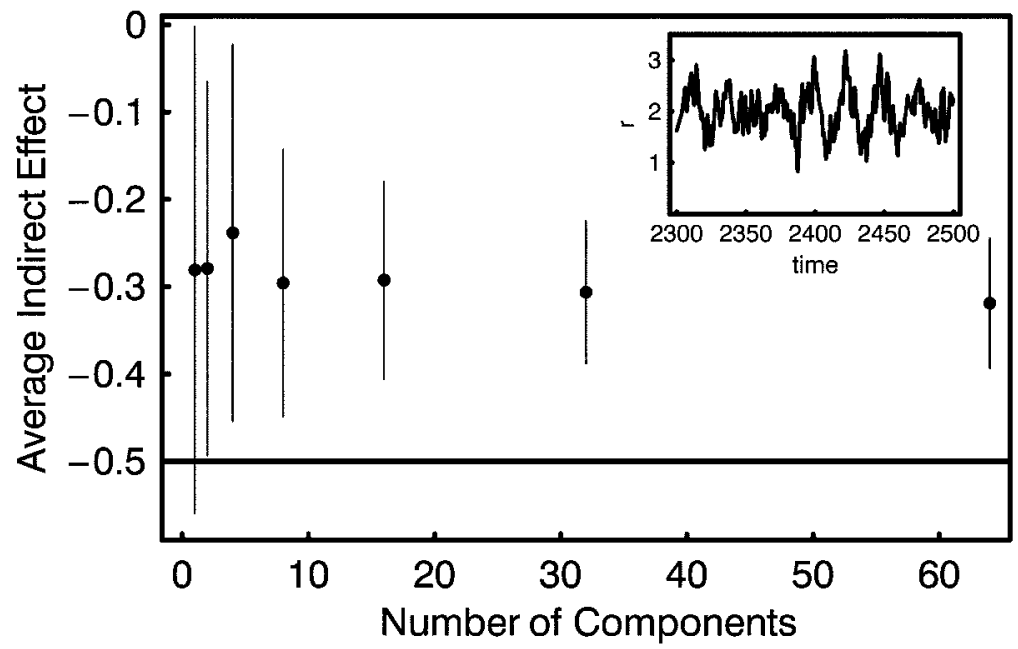

Figure 5: Indirect effect between prey populations in a model with realistic environmental variation. The model and parameters are the same as in figure 3 with variation in the prey growth rate $r$. Environmental variation is modeled as pink noise with components chosen across a range of periods from 1 to 30 . The mean is plotted from 25 random draws. $a$, Average indirect effect between prey is plotted versus the dominant forcing period. Environmental variation consists of a dominant period as listed on the axis and 64 additional pink-noise periods. The amplitude of the dominant period is 10 times greater than it would be based on pink noise. Inset is example of environmental variation with dominant period of 10. $b$, Average indirect effect is plotted versus the number of components of environmental variation. The amplitudes of all components conform to pink noise. Bars represent standard deviations. Inset is example of 64-component environmental variation.

direct effects occur when the period of environmental variation is shorter than the natural period of the system.

Temporal variation does more than just reduce the strength of interactions. Schoener (1993) argues that indirect effects would be weakened by stochastic variation. $\mathrm{He}$ argues that periods of low abundance of one or more intermediate populations are likely to interrupt a chain of indirect interactions. However, the analysis presented here does not provide clear support for Schoener's idea. It is not always true that variation reduces the strength of indirect interactions via a shared predator in the case of exogenous variation. In some scenarios, such as that described in figure 5, variation does tend to decrease interaction strengths, but it is misleading to conceptualize the effect of variation as increasing or decreasing the magnitude of a defined effect because variation is capable of 
changing the sign of indirect effects. The effect of fluctuations depends on details of the variation and the model; a similar conclusion was reached by Holt and Barfield (2003) when examining coexistence in an open system. There may be generalizations that emerge, but these will require analysis of many more types of food webs than the simple one considered here.

While the focus of this article has been on models that reach a stable equilibrium in the absence of environmental variation, environmental variation also can alter indirect interactions in models that maintain sustained periodic cycles in the absence of environmental variation. Environmental variation can increase or decrease the magnitude of indirect effects. For example, when examining parameters with large enough handling times to generate endogenous cycles, but for which indirect interactions are still negative, exogenous variation can interact with the cycling dynamics and generate positive effects in the same manner that exogenous variation amplified the damped cycles (C. E. Brassil, unpublished data).

Resonance between environmental variation and the populations was important in modifying indirect effects. A number of models have been fit to time series of real populations, and it has been found that resonance is important in understanding many population cycles (Leirs et al. 1997; Lima et al. 1999; Rohani et al. 2002; Murua et al. 2003; Koelle and Pascual 2004). The population dynamics of rice rats in Chile is best explained by the combination of a deterministic model and climate variation represented by the Southern Oscillation Index and the Antarctic Oscillation Index (Murua et al. 2003). Fairly regular and wide-reaching environmental cycles like the El Niño Southern Oscillation may provide regular forcing that can approximate the effects modeled here by deterministic sinusoidal variation in growth parameters. At shorter time scales, seasonal change is a ubiquitous form of environmental variation.

Environmental variation and resonance have important consequences for disease models (Greenman et al. 2004; Ireland et al. 2004). Evolution of season-specific pathogen strains has been suggested as important in understanding cholera outbreaks in Bangladesh (Koelle et al. 2005). Extensions of the work presented here could consider how the adaptation of traits in fluctuating environments might modify indirect interactions.

The fact that exogenous and endogenous variation can have similar effects in single-species models suggested that previous results demonstrating positive effects due to endogenous variation might also apply to exogenous variation. Here, I have shown that this speculation is true in some cases and becomes increasingly common in highly nonlinear models. Positive indirect effects between two prey that share a predator appear to be relatively common when cycles are generated by endogenous mechanisms (Abrams et al. 1998; Brassil and Abrams 2004). It is impossible to estimate the likelihood of exogenous variation leading to large changes in indirect effects. Endogenous fluctuations by definition correspond to the natural frequencies of the biological system, and there is no a priori reason why environmental forcing should be close to the natural frequency. However, exogenous variation in nonlinear models can lead to large changes in indirect effects, often generating positive effects over a relatively wide range of periodicities. The question remains as to why there continues to be no good empirical example of long-term, positive indirect effects in the empirical literature.

\section{Acknowledgments}

I would like to thank P. A. Abrams, A. F. Agrawal, J. Besch, H. Cyr, R. M. Nisbet, and B. Shuter for comments on this work. This work was supported in part by an Ontario Graduate Scholarship, in part by individual research grant from the Natural Science and Engineering Research Council of Canada to P. A. Abrams, and in part by a grant from the Andrew W. Mellon Foundation to K. L. Gross. This article was based on work submitted to the University of Toronto in partial fulfillment of the $\mathrm{PhD}$ degree. This is Kellogg Biological Station contribution 1183.

\section{Literature Cited}

Abrams, P. A. 1987. Indirect interactions between species that share a predator: varieties of indirect effects. Pages 38-54 in W. C. Kerfoot and A. Sih, eds. Predation: direct and indirect impacts on aquatic communities. University Press of New England, Dartmouth, NH.

. 1997. Variability and adaptive behavior: implications for interactions between stream organisms. Journal of the North American Benthological Society 16:358-374.

- 2004. When does periodic variation in resource growth allow robust coexistence of competing consumer species? Ecology 85: 372-382.

Abrams, P. A., and H. Matsuda. 1996. Positive indirect effects between prey species that share predators. Ecology 77:610-616.

Abrams, P. A., R. D. Holt, and J. D. Roth. 1998. Apparent competition or apparent mutualism? shared predation when populations cycle. Ecology 79:201-212.

Brassil, C. E., and P. A. Abrams. 2004. The prevalence of asymmetrical indirect effects in two-host-one-parasitoid systems. Theoretical Population Biology 66:71-82.

Chaneton, E. J., and M. B. Bonsall. 2000. Enemy-mediated apparent competition: empirical patterns and the evidence. Oikos 88:380394.

Coulson, T., P. Rohani, and M. Pascual. 2004. Skeletons, noise and population growth: the end of an old debate? Trends in Ecology \& Evolution 19:359-364.

Dushoff, J., J. B. Plotkin, S. A. Levin, and D. J. D. Earn. 2004. Dynamical resonance can account for seasonality of influenza ep- 
idemics. Proceedings of the National Academy of Sciences of the USA 101:16915-16916.

Ellner, S., and P. Turchin. 1995. Chaos in a noisy world: new methods and evidence from time-series analysis. American Naturalist 145: 343-375.

Greenman, J., M. Kamo, and M. Boots. 2004. External forcing of ecological and epidemiological systems: a resonance approach. Physica D: Nonlinear Phenomena 190:136-151.

Greenman, J. V., and T. G. Benton. 2003. The amplification of environmental noise in population models: causes and consequences. American Naturalist 161:225-239.

Heimpel, G. E., C. Neuhauser, and M. Hoogendoorn. 2003. Effects of parasitoid fecundity and host resistance on indirect interactions among hosts sharing a parasitoid. Ecology Letters 6:556-566.

Holt, R. D. 1977. Predation, apparent competition, and the structure of prey communities. Theoretical Population Biology 12:197-229.

Holt, R. D., and M. Barfield. 2003. Impacts of temporal variation on apparent competition and coexistence in open ecosystems. Oikos 101:49-58.

Holt, R. D., and J. H. Lawton. 1993. Apparent competition and enemy-free space in insect host-parasitoid communities. American Naturalist 142:623-645.

Ireland, J. M., R. A. Norman, and J. V. Greenman. 2004. The effect of seasonal host birth rates on population dynamics: the importance of resonance. Journal of Theoretical Biology 231:229-238.

Kendall, B. E., J. Prendergast, and O. N. Bjørnstad. 1998. The macroecology of population dynamics: taxonomic and biogeographic patterns in population cycles. Ecology Letters 1:160-164.

Koelle, K., and M. Pascual. 2004. Disentangling extrinsic from intrinsic factors in disease dynamics: a nonlinear time series approach with an application to cholera. American Naturalist 163: 901-913.

Koelle, K., M. Pascual, and M. Yunus. 2005. Pathogen adaptation to seasonal forcing and climate change. Proceedings of the Royal Society of London B 272:971-977.

Leirs, H., N. C. Stenseth, J. D. Nichols, J. E. Hines, R. Verhagen, and W. Verheyen. 1997. Stochastic seasonality and nonlinear density- dependent factors regulate population size in an African rodent. Nature 389:176-180.

Lima, M., J. E. Keymer, and F. M. Jaksic. 1999. El Niño-southern oscillation-driven rainfall variability and delayed density dependence cause rodent outbreaks in western South America: linking demography and population dynamics. American Naturalist 153: 476-491.

Murua, R., L. A. Gonzalez, and M. Lima. 2003. Population dynamics of rice rats (a hantavirus reservoir) in southern Chile: feedback structure and non-linear effects of climatic oscillations. Oikos 102: 137-145.

Nisbet, R. M., and W. S. C. Gurney. 1982. Modelling fluctuating populations. Wiley, New York.

Puccia, C. J., and R. Levins. 1985. Qualitative modeling of complex systems: an introduction to loop analysis and time averaging. Harvard University Press, Cambridge, MA.

Rinaldi, S., S. Muratori, and Y. Kuznetsov. 1993. Multiple attractors, catastrophes and chaos in seasonally perturbed predator prey communities. Bulletin of Mathematical Biology 55:15-35.

Rohani, P., M. J. Keeling, and B. T. Grenfell. 2002. The interplay between determinism and stochasticity in childhood diseases. American Naturalist 159:469-481.

Ruel, J. J., and M. P. Ayres. 1999. Jensen's inequality predicts effects of environmental variation. Trends in Ecology \& Evolution 14: 361-366.

Schoener, T. W. 1993. On the relative importance of direct versus indirect effects in ecological communities. Pages 365-411 in $\mathrm{H}$. Kawanabe, J. E. Cohen, and K. Iwasaki, eds. Mutualism and community organization: behavioural, theoretical, and food-web approaches. Oxford University Press, Oxford.

Vasseur, D. A., and P. Yodzis. 2004. The color of environmental noise. Ecology 85:1146-1152.

Webster, M. S., and G. R. Almany. 2002. Positive indirect effects in a coral reef fish community. Ecology Letters 5:549-557.

Associate Editor: Will Wilson Editor: Donald L. DeAngelis 